Regular Article

\title{
Discontinuous strain-induced martensite transformation related to the Portevin-Le Chatelier effect in a medium manganese steel
}

Binhan Sun ${ }^{\mathrm{a}, *}$, Nicolas Vanderesse ${ }^{\mathrm{b}}$, Fateh Fazeli ${ }^{\mathrm{c}}$, Colin Scott $^{\mathrm{c}}$, Jianqiang Chen ${ }^{\mathrm{b}}$, Philippe Bocher ${ }^{\mathrm{b}}$, Mohammad Jahazi ${ }^{\text {b }}$, Stephen Yue ${ }^{\mathrm{a}}$

a Department of Materials Engineering, McGill University, 3610 University Street, Montreal, QC H3A 0C5, Canada

b Department of Mechanical Engineering École de Technologie Supérieure, 1100 Notre-Dame Ouest Street, Montreal, QC H3C 1 K3, Canada

c CanmetMATERIALS, Natural Resources Canada, 183 Longwood Road South, Hamilton, ON, L8P OA5, Canada 
Discontinuous strain-induced martensite transformation related to the

Portevin-Le Chatelier effect in a medium manganese steel

Binhan Sun ${ }^{\mathrm{a} *}$, Nicolas Vanderesse ${ }^{\mathrm{b}}$, Fateh Fazeli ${ }^{\mathrm{c}}$, Colin Scott ${ }^{\mathrm{c}}$, Jianqiang Chen $^{\mathrm{b}}$, Philippe Bocher ${ }^{\mathrm{b}}$, Mohammad Jahazi ${ }^{\mathrm{b}}$, and Stephen Yue ${ }^{\mathrm{a}}$

${ }^{\mathrm{a}}$ Department of Materials Engineering, McGill University, 3610 University Street, Montreal, QC, H3A 0C5, Canada;

${ }^{b}$ Department of Mechanical Engineering, École de technologie supérieure, 1100 Notre-Dame Ouest Street, Montreal, QC, H3C 1K3, Canada;

${ }^{\mathrm{c} C a n m e t M A T E R I A L S, ~ N a t u r a l ~ R e s o u r c e s ~ C a n a d a, ~} 183$ Longwood Road South, Hamilton, ON, L8P 0A5, Canada.

*Corresponding author: Binhan Sun,

Tel: +1-514-431-8181,

Email: binhan.sun@mail.mcgill.ca.

\begin{abstract}
:
The correlation between the strain-induced martensite transformation (SIMT) and the Portevin-Le Chatelier (PLC) effect during tensile testing of a ferrite-austenite duplex medium manganese (Mn) steel was investigated in this study. A discontinuous SIMT phenomenon (D-SIMT) was observed in the steel by in situ magnetic induction measurements complemented with ex situ X-ray diffraction analysis. This unique behavior was due to the localized martensite formation in the PLC bands forming and propagating continuously during tensile straining. The PLC bands were characterized by the digital image correlation technique and their origin is further discussed.
\end{abstract}

Keywords: medium Mn steels; strain-induced martensite transformation; Portevin-Le Chatelier (PLC) effect; digital image correlation. 
Strain-induced martensite transformations have been used for some time in designing modern advanced high strength steels (AHSS), such as the low alloy transformation-induced plasticity (TRIP) [1], carbide-free bainite (CFB) [2], and quenching and partitioning (Q\&P) [3] steels, due to its role in the enhancement of work hardening and ductility. More recently, medium Mn steels containing 3 to $10 \mathrm{wt} . \%$ Mn were developed [4-8]; the higher Mn content ensures a large fraction of metastable retained austenite available to transform during room temperature deformation, which is the main contributor to the excellent strength-ductility balance of such steels. It is well established that plastic deformation provides new nucleation sites which consist of $\varepsilon$-martensite, stacking fault bundles and mechanical twins [9, 10], for $\alpha^{\prime}$-martensite embryos. As such, the amount of $\alpha^{\prime}$-martensite continuously increases with plastic strain at constant temperature; the overall transformation kinetics normally shows a sigmoidal shape [5, 9]. However, this relation is expected to be changed when the steels undergo inhomogeneous deformation.

It was remarked in the literature $[5,11]$ that some plastic instability phenomena such as the Lüders banding and PLC effect, may occur in medium Mn steels. The latter is generally characterized by serrated plastic flow in the tensile stress-strain curves and spatio-temporal organized deformation bands. The PLC effect is also commonly observed in ferritic steels [12] and austenitic high Mn twinning-induced plasticity (TWIP) steels $[13,14]$ tested at various temperatures and strain rates. Generally, it is proposed to be associated with dynamic strain ageing (DSA), which arises from the dynamic interaction between mobile dislocations and diffusing solute atoms. However, the PLC phenomenon in medium Mn steels is expected to be more complicated due to the multiphase structure and the strain-induced transformation feature; its origin and the correlation with the SIMT in such steels have rarely been studied. To this end, the present work investigates the relationship between the PLC phenomenon and SIMT in a ferrite-austenite duplex medium $\mathrm{Mn}$ steel, by a combination of digital image correlation and in situ magnetic induction measurements complemented with ex situ $\mathrm{X}$-ray diffraction analysis.

The chemical composition of the investigated steel is Fe-0.2C-10.3Mn-2.9Al (in wt.\%). The ingot was prepared in a vacuum induction furnace, reheated to $1230{ }^{\circ} \mathrm{C}$ and hot rolled to $\sim 3 \mathrm{~mm}$ plates, followed by air cooling to room temperature, producing a predominately martensitic structure. The hot rolled plates were heat 
treated at $500{ }^{\circ} \mathrm{C}$ for $2 \mathrm{~h}$ in order to temper the martensite phase, after which cold rolling was conducted with a thickness reduction of $\sim 50 \%$. Samples were then intercritically annealed at 700 and $750{ }^{\circ} \mathrm{C}$ for $5 \mathrm{~min}$ followed by water quenching, in order to produce various austenite conditions; they are referred to as IA-700 and IA-750 in this study for convenience. Room temperature uniaxial tensile tests were carried out on the annealed specimens at a constant crosshead speed corresponding to a macroscopic engineering strain rate of $6 \times 10^{-4} \mathrm{~s}^{-1}$. The tensile samples were machined according to ASTM E-8 subsize standard (25 mm gage length) and oriented parallel to the rolling direction. Microstructure observations were performed with a field-emission scanning electron microscope (FE-SEM, FEI Inspect F-50). The change in the fraction of retained austenite due to $\alpha$-martensite formation during tensile tests was analyzed by an in situ magnetic induction method implemented by a Feritscope (Fischer, FMP 30), combined with ex situ X-ray diffraction (XRD) analysis; the analyzed surface areas of the Feritscope and XRD were a $2 \mathrm{~mm}$ diameter circle and a $4.8 \times 4.8 \mathrm{~mm}$ square, respectively. The probe of the Feritscope was tightly attached to the center of the tensile samples during the tensile testing, and the value was recorded every $2.5 \mathrm{~s}$. Since the Feritscope was initially designed to measure the ferrite content in austenitic steels, it was calibrated in advance by comparing with the values generated by XRD on several prior cold rolled IA-750 samples. A linear relationship with a Pearson correlation coefficient of 0.994 exists between the two measurements, as was observed by other researchers [10]. Digital image correlation (DIC) was used to investigate the localized deformation during tensile testing. Images were acquired with a G-504 Manta digital camera (Allied Vision Technologies GmbH, Stadtroda, Germany) with a telecentric lens at a rate of 9 frames per second. A field of view of $\sim 14 \times 5 \mathrm{~mm}^{2}$ was characterized with numerical resolution of $7 \mu \mathrm{m} /$ pixel. The strain fields were computed using the in-house OpenDIC software [15] and post-processed with the image analysis program Fiji [16].

Fig. 1 shows the microstructures of the IA-700 and IA-750 sample, consisting of only ferrite $(\alpha)$ and austenite $(\gamma)$ with sub-micron grains. The ultra-fine grain size is due to the phase transformation from martensite and static recrystallization concurrently taken place during intercritical annealing [6]. The measured fraction of austenite and its equilibrium composition calculated by FactSage 6.4 [17] with the FSstel database in the two samples are shown in Table 1. It is obvious that the higher annealing temperature increases austenite fraction but decreases austenite $\mathrm{C}$ and $\mathrm{Mn}$ content; 
thus, austenite stability is reduced at higher temperatures, as also indicated from the higher $\mathrm{Md}_{30}$ temperature (the temperature at which half of the austenite is transformed to martensite after a strain of 30\%) of the IA-750 sample. The lower C and Mn level of the austenite in the IA-750 sample also results in a lower stacking fault energy (SFE) compared with IA-700.

Table 1 Fraction and equilibrium composition of austenite in the two samples

\begin{tabular}{|c|c|c|c|c|c|c|}
\hline Sample & $\begin{array}{c}\mathrm{C} \\
(\text { FactS }\end{array}$ & & & $\operatorname{Md}_{30}\left({ }^{\circ} \mathrm{C}\right) *$ & $\begin{array}{c}\text { Austenite SFE } \\
\left(\mathrm{mJ} / \mathrm{m}^{2}\right) * *\end{array}$ & $\begin{array}{c}\text { Austenite Fraction } \\
\text { (XRD, wt. \%) }\end{array}$ \\
\hline IA-700 & 0.32 & 13.1 & 2.5 & 289.2 & 18.2 & $33.1(1.5)$ \\
\hline IA-750 & 0.26 & 11.6 & 2.7 & 334.9 & 14.4 & $46.7(2.5)$ \\
\hline \multicolumn{7}{|c|}{$\begin{array}{l}* \mathrm{Md}_{30}\left({ }^{\circ} \mathrm{C}\right)=608-515 C(w t . \%)-12 M n(w t . \%)[18] ; \\
* * \text { Austenite SFE was calculated by the thermodynamic model }: \gamma_{\mathrm{SFE}}=2 \rho \Delta G^{\gamma \rightarrow \varepsilon}+2 \sigma_{\gamma / \varepsilon} \text {, where } \rho \text { is } \\
\text { the molar density along }\{111\} \text { planes, } \Delta G^{\gamma \rightarrow \varepsilon} \text { is the molar Gibbs energy of the } \gamma \text { to } \varepsilon \text { phase } \\
\text { transformation, and } \sigma_{\gamma / \varepsilon} \text { is the interfacial energy, selected to be } 5 \mathrm{~mJ} / \mathrm{m}^{2} . \text { The detailed calculation } \\
\text { method was described in Curtze's work [19], and the thermodynamic data was selected from the } \\
\text { references [19-23]. }\end{array}$} \\
\hline
\end{tabular}

The true stress-strain curve and the work hardening rate of the two samples are shown in Figs. 2 (a) and (b). Two distinct flow behaviors can be observed: firstly, the IA-750 sample shows an obvious serrated flow phenomenon, beginning very early after the Lüders strain, whereas no distinct serrations can be observed for the IA-700 sample. Secondly, the IA-750 sample exhibits a much higher rate of work hardening $(d \sigma / d \varepsilon)$ than the IA-700 and a larger elongation. The work hardening rate of the IA-750 sample, in general, first increases up to about $4000 \mathrm{MPa}$ and then decreases after about 0.1 strain; however, the IA-700 sample shows a monotonic decrease after yielding. Several oscillations were observed in the work hardening curve of IA-750 (Fig. 2 (b)), which were associated with the serrations of the flow curve and the numerical derivative. Fig. 2 (c) presents the change of transformed austenite fraction during tensile tests, which has been normalized by the initial austenite fraction $\left(\Delta \gamma / \gamma_{0}\right)$. The change of austenite fraction is due to the $\alpha$ '-martensite transformation; no $\varepsilon$-martensite was observed after deformation either from the microstructure or the XRD patterns. The value confirms a much more pronounced rate of SIMT (i.e. lower austenite stability) in the IA-750 sample, which is believed to be the main reason for the higher work hardening. However, the SIMT in the IA-750 sample is characterized by a distinct discontinuous transformation behavior including consecutive pairs of martensite bursts and plateaus. This D-SIMT phenomenon was also confirmed by the 
XRD experiments, which were carried out on one tensile specimen characterized at very small strain intervals of $\sim 0.005$. The fact that this phenomenon only occurs in the IA-750 sample but not the IA-700 sample suggests that it is most likely a material feature pertaining to the composition and stability of austenite.

It should be noted that this D-SIMT phenomenon has not been observed with in situ phase quantification methods in conventional TRIP steels [24] or similar medium Mn steels $[5,6]$. The first martensite burst in the IA-750 sample in Fig. 2 (c) was most likely due to the localized SIMT inside the Lüders band, as reported by other researchers $[11,25]$, however, in contrast to the current study, the Lüders strain in their work was abnormally large such that the SIMT was almost saturated after the Lüders strain. Some SIMT models such as Burke-Matsumura and Olson-Cohen (OC) models [5,9] also failed to explain this discontinuous behavior in the investigated alloy. However, the overall trend of the SIMT in the IA-750 specimen still follows the sigmoidal shape predicted by the OC model; the curve fitting, based on the OC model, yields the following relations between the fraction of martensite and global plastic strain: $f_{\alpha^{\prime}}=\left[1-\exp \left[-1.25 \times[1-\exp (-11.1 \varepsilon)]^{1.51}\right] \times f_{\gamma}\right.$, where $f_{\gamma}$ is the initial austenite fraction before deformation. In order to explain this unique behavior, the strain localization in the IA-750 sample during tensile tests was investigated by DIC. Fig. 3 (a) shows the average engineering strain in the DIC analyzed area as a function of deformation time, and several strain maps corresponding to the propagation of two successive PLC bands. The strain behavior oscillates between strain increases and plateaus. It can be clearly observed that the passage of PLC bands in the DIC analyzed area results in strain increases, whereas the plateaus on the strain-time curve indicate that the banding behavior occurs outside of the detected area. The angle between the direction of the bands and the tensile axis is $\sim 64^{\circ}$, which is larger than that reported in TWIP steels $\left(\sim 55^{\circ}\right)$ [14]. The corresponding strain profile of the two bands along the tensile axis is plotted in Fig. 3 (b), from which the band strain can be estimated to be 0.013 and 0.018 for the $1^{\text {st }}$ and $2^{\text {nd }}$ band, respectively. Such local strains would result in around $2.3 \%$ martensite formation, calculated based on the OC model for the IA-750 sample by taking into account the local strain before and after the bands propagation. This number is very similar to the values of the martensite bursts ( $2 \%$ in average) in the IA-750 sample detected by Feritscope in Fig. 2 (c) (excluding the first one which is due to the Lüders band). This is the first indication that localized SIMT occurs in the PLC bands and moves following the bands 
propagation.

The macroscopic engineering strain measured by the extensometer and the normalized austenite transformed fraction of the IA-750 sample as a function of deformation time in the plastic deformation range is shown in Fig. 4 (a). Ten distinct plateaus were observed and marked in the strain-time curve, indicating that ten PLC bands were initiated and propagated afterwards during tensile straining [14]. The same number of steps was also observed in the austenite transformed fraction curve. This also strongly suggests that the mechanism of D-SIMT correlates with the PLC bands, namely martensite forms locally in the PLC bands. Similar D-SIMT behavior was also reported by Hedström [26] in a metastable austenite stainless steel using in situ high-energy X-ray diffraction techniques, although the strain localization was not investigated and no detailed explanation was given. However, it was found in his work that the discontinuous transformation event correlates with the stepwise increased lattice strains and peak broadening of the austenite phase, which would essentially occur if deformation bands propagate through the XRD detected area. Therefore, it is probable that the bursts of martensite fraction were derived from the bands passing through the austenite measurement range, followed by plateaus when the band fronts left the detected area. This idea can be verified by calculating the propagation velocity of the deformation bands, separately using DIC, the macroscopic strain-time curve and the transformed austenite-time curve in Fig. 4 (a). The propagation velocity can be calculated using the following equation: $v_{\mathrm{p}}=L / \Delta t$, where $L$ is the propagation length or distance, depending on the probed area of the detector ( $25 \mathrm{~mm}$ for the extensometer and $2 \mathrm{~mm}$ for the Feritscope), and $\Delta t$ is the bands propagation time inside the detected areas, which can be read directly from the strain maps, and the two curves (the magnified part of the two curves as illustrated by the inset figure in Fig. 4 (a)). The average propagation speeds determined from DIC, the strain-time data and the austenite-time data are around $0.6 \mathrm{~mm} / \mathrm{s}, 0.8 \mathrm{~mm} / \mathrm{s}$ and 0.7 $\mathrm{mm} / \mathrm{s}$, respectively, which are quite comparable. These close values confirm the localized martensite formation in the propagating deformation bands occurred continuously during straining.

The dual phase nature of the investigated steel (ferrite-austenite) increases the complexity of interactions between the possible ongoing mechanisms. PLC bands are generally observed in ferritic structures upon straining of specimens at elevated 
temperatures where a sufficient diffusivity of solutes to trigger DSA is ensured [12]. However, austenitic TWIP steels can show DSA in conjunction with PLC effects at room temperature, which is very likely related to their high Mn content and low SFE $[13,14]$. Considering the similarity in austenite characteristics between the investigated alloy and TWIP steels, it is not surprising to observe the PLC phenomenon in the present study which could most likely stem from the plastic instability of the austenite phase. In fact, the essential condition for the occurrence of localized SIMT in the PLC bands is the sufficient local deformation of austenite. However, the origin of the observed PLC effect in this study is more complex due to the fact that they correlate with the martensite transformation. Hence, the interplay between the SIMT and the PLC phenomenon is valuable to be discussed.

The engineering strain and stress as a function of deformation time for the IA-750 sample is shown in Fig. 4 (b), in which serrations in the stress curve can be clearly seen when the PLC bands are initiated and traverse the gage length. It appears that there is a localized SIMT associated with PLC bands, which could probably contribute to the overall work hardening but not the sudden intermittent rises in the overall flow stress. The subsequent small stress drops observed in Fig. 4 (b) are also difficult to explain by SIMT. The fact that the IA-700 sample and other medium Mn steels with similar or even higher fractions of SIMT [5, 6] compared with IA-750 do not show this noticeable serrated flow suggests that the discontinuous SIMT is an outcome of the PLC bands passage but not the reason for the observed PLC behavior and the jerky flow. On the other hand, the classical DSA theories initially proposed by Cottrell [27] and further developed by other researchers [28, 29] can well explain these serrations; it is postulated that mobile dislocations can temporarily be pinned by interstitial carbon atoms diffusing towards the dislocation cores. During the pinning time, the flow stress increases until it reaches a critical unpinning value which allows the dislocations to break away and move at high velocities, resulting in apparent drops of the flow stress. In TWIP steels, the estimated bulk diffusivity of $\mathrm{C}$ atoms at room temperature is too low to arrest dislocations for conventional DSA to occur [13]. The calculated thermal activation energy for PLC in TWIP steels is an order of magnitude lower than that for bulk $\mathrm{C}$ diffusion [30]. Note that the activation energy determined by conventional analysis for PLC could be inaccurate, due to the imprecise procedure to determine the critical strain for the onset of PLC bands [31]. Further, it is difficult to deconvolute the contribution of dislocation glide, twining and martensite formation 
to the occurrence of PLC in high/medium Mn steels, thus a conclusive estimation of the activation energy for the observed serrated flow might not be trivial. Nevertheless, the DSA effect in TWIP steels was therefore proposed to be associated with carbon reorientation between different interstitial lattice sites along with the interaction between C-Mn complexes and partial dislocations/stacking faults [13, 14]. The activation energy for the required single atomic jump of $\mathrm{C}$ inside the fault region between octahedral and tetrahedral sublattice sites was estimated to be around $0.75 \mathrm{eV}$ [13], which is significantly lower than the determined value $(1.78 \mathrm{eV})$ for $\mathrm{C}$ bulk diffusion in high Mn steels [32]. Higher $\mathrm{C}$ and Mn contents increase the probability of C-Mn complexes formation, which tends to impede the glide of dislocation lines thus facilitates the occurrence of DSA. However, the increased SFE due to C and Mn enrichment in austenite decreases the density of partial dislocations and stacking faults, which diminishes the intensity of DSA. As such the serrated flow could be seen merely within a suitable range of austenite compositions. For example, strain instability and tangible serrated flow were not found in the IA-700 sample regardless of its higher $\mathrm{C}$ and $\mathrm{Mn}$ contents in austenite. It has to be noted that in contrast to IA-750 and due to a lower intercritical annealing temperature, the IA-700 sample was not fully recrystallized, which could potentially influence the observed PLC response; this requires future studies. In addition, the counter effect of martensite formation on PLC in the investigated alloy is also suggested here in terms of: (1) Mobile dislocations might be additionally pinned by the small martensite embryos until a higher stress is reached to unpin the dislocations. In order to maintain the martensite-induced pinning and associated unpinning process, enough numbers of martensite embryos should be continuously transformed, which is possible for the IA-750 sample due to its lower austenite stability; (2) higher amounts of martensite transformation might have a suppression effect on DSA associated with the trapping of trailing partials [33]. These two conflicting effects might further explain why the PLC effect and D-SIMT only occur in a certain range of austenite stability and would disappear in the samples with a higher amount of SIMT (even with lower SFE), which is evidenced in the work of other researchers [5].

In summary, the correlation between the SIMT and strain instability (the PLC effect) in a ferrite-austenite duplex medium Mn steel during tensile tests was investigated, using in situ magnetic induction measurements combined with ex situ XRD analysis and DIC techniques. It was shown that the SIMT in the IA-750 sample behaved 
discontinuously, which was due to the localized martensite formation within the PLC bands nucleating intermittently and propagating continuously during tensile straining.

The authors gratefully acknowledge Dr. H. Monajati from Department of Mechanical Engineering, École de technologie supérieure, for the support on the Feritscope measurement. One of the authors (Binhan Sun) acknowledges with gratitude the scholarship support received from China Scholarship Council (CSC) and the McGill Engineering Doctoral Award (MEDA) program. 


\section{Reference}

[1] P.J. Jacques, Phase transformations in transformation induced plasticity (TRIP)-assisted multiphase steels, in: E. Pereloma and D.V. Edmonds (Eds.), Phase Transformations in Steels, Vol. 2, Woodhead Publishing Ltd., Philadelphia, 2012.

[2] C. García-Mateo, F.G. Caballero, Mater. Trans. 46 (2005) 1839-1846.

[3] J.G. Speer, Phase transformations in quenched and partitioned steels, in: E. Pereloma and D.V. Edmonds (Eds.), Phase Transformations in Steels, Vol. 2, Woodhead Publishing Ltd., Philadelphia, 2012.

[4] B. Sun, H. Aydin, F. Fazeli, S. Yue, Metall. Mater. Trans. A 47 (2016) 1782-1791.

[5] P.J. Gibbs, E. De Moor, M.J. Merwin, B. Clausen, J.G. Speer, D.K. Matlock, Metall. Mater. Trans. A 42 (2011) 3691-3702.

[6] J. Han, S.J. Lee, J.G. Jung, Y.K. Lee, Acta Mater. 78 (2014) 369-377.

[7] B. Sun, F. Fazeli, C. Scott, X. Yan, Z. Liu, X. Qin, S. Yue, Scripta Mater. 130 (2017) 49-53.

[8] B. Sun, F. Fazeli, C. Scott, S. Yue, Metall. Mater. Trans. A 47 (2016) 4869-4882.

[9] G.B. Olson, M. Cohen, Metall. Trans. A 6 (1975) 791-795.

[10] J. Talonen, P. Aspegren, H. Hänninen, Mater. Sci. Technol. 20 (2004) 1506-1512.

[11] X. Wang, L. Wang, M. Huang, Acta Mater. 124 (2017) 17-29.

[12] A.K. Sachdev, Metall. Trans. A 13 (1982) 1793-1797.

[13] S.J. Lee, J. Kim, S.N. Kane, B.C. De Cooman, Acta Mater. 59 (2011) 6809-6819.

[14] L. Chen, H.S. Kim, S.K. Kim, B.C. De Cooman, ISIJ Int. 47 (2007) 1804-1812.

[15] N. Vanderesse, M. Lagacé, F. Bridier, P. Bocher, Microsc. Microanal. 19 (2013) 820-821.

[16] J. Schindelin, I. Arganda-Carreras, E. Frise, V. Kaynig, M. Longair, T. Pietzsch, S. Preibisch, C. Rueden, S. Saalfeld, B. Schmid, J. Tinevez, D. J. White, V. Hartenstein, K. Eliceiri, P. Tomancak, A. Cardona, Nat. methods 9 (2012) 676-682.

[17] C.W. Bale, E. Bélisle, P. Chartrand, S.A. Decterov, G. Eriksson, K. Hack, I.H. Jung, Y.B. Kang, J. Melançon, A.D. Pelton, C. Robelin, S. Petersen, Calphad 33 (2009) 295-311.

[18] T.H. Lee, C.S. Oh, S.J. Kim, Scripta Mater. 58 (2008) 110-113.

[19] S. Curtze, V.T. Kuokkala, Acta Mater. 58 (2010) 5129-5141.

[20] A.T. Dinsdale, Calphad 15 (1991) 317-425.

[21] A. Dumay, J.P. Chateau, S. Allain, S. Migot, O. Bouaziz, Mater. Sci. Eng., A 483 (2008) 184-187.

[22] S. Allain, J.P. Chateau, O. Bouaziz, S. Migot, N. Guelton, Mater. Sci. Eng., A 387 
(2004) 158-162.

[23] O. Grässel, G. Frommeyer, C. Derder, H. Hofmann, J. Phys. IV France 7 (1997) 383-388.

[24] S. Brauser, A. Kromm, Th. Kannengiesser, M. Rethmeier, Scripta Mater. 63 (2010) 1149-1152.

[25] J.H. Ryu, J.I. Kim, H.S. Kim, C.S. Oh, H.K.D.H. Bhadeshia, D.W. Suh, Scripta Mater. 68 (2013) 933-936.

[26] P. Hedström, U. Lienert, J. Almer, M. Odén, Scripta Mater. 56 (2007) 213-216.

[27] A.H. Cottrell, B.A. Bilby, Proceedings of the Physical Society. Section A 62 (1949) 49-62.

[28] A. Van den Beukel, Physica status solidi (a) 30 (1975) 197-206.

[29] P. McCormigk, Acta Metall. 20 (1972) 351-354.

[30] S. Allain, P. Cugy, C. Scott, J.P. Chateau, A. Rusinek, A. Deschamps, Int. J. Mater. Res. 99 (2008) 734-738.

[31] A. Roth, T.A. Lebedkina, M.A. Lebyodkin, Mater. Sci. Eng., A 539 (2012) 280-284.

[32] T. Shun, C.M. Wan, J.G. Byrne, Acta Metall. et Mater. 40 (1992) 3407-3412.

[33] M. Koyama, T. Sawaguchi, K. Tsuzaki, Philos. Mag. A 92 (2012) 3051-3063. 

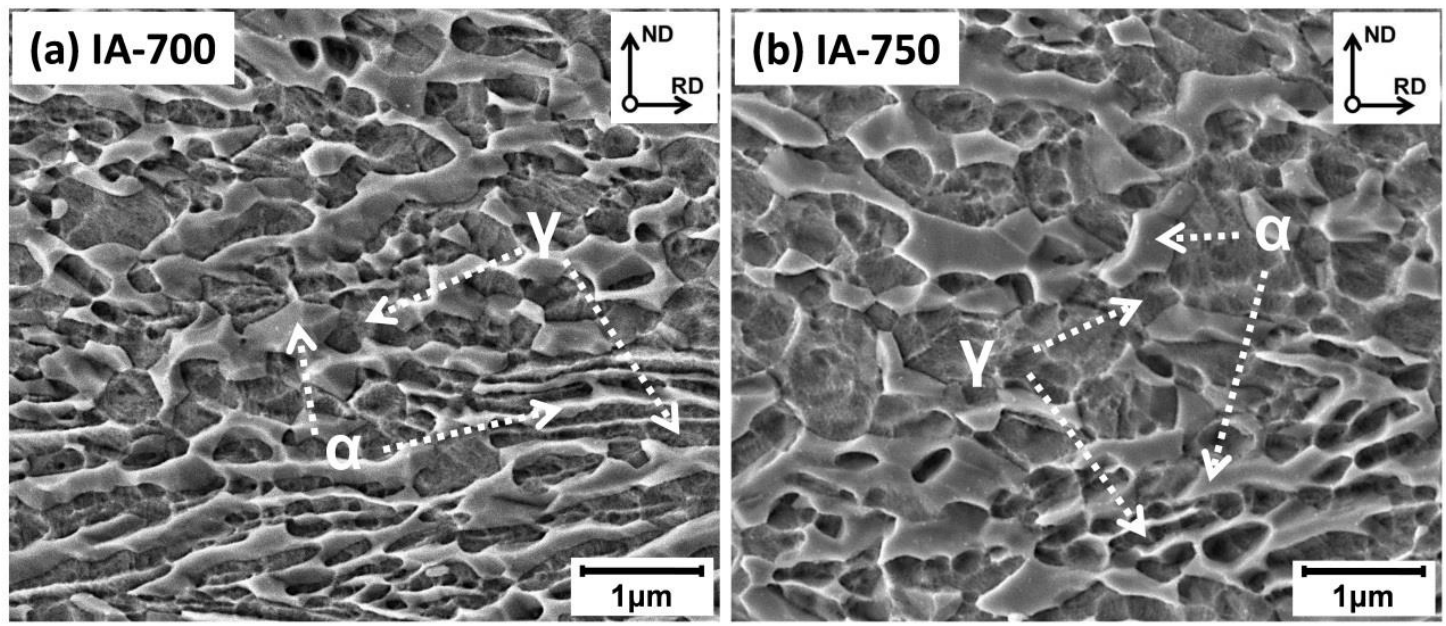

Fig. 1 SEM micrographs of the (a) IA-700 and (b) IA-750 sample.
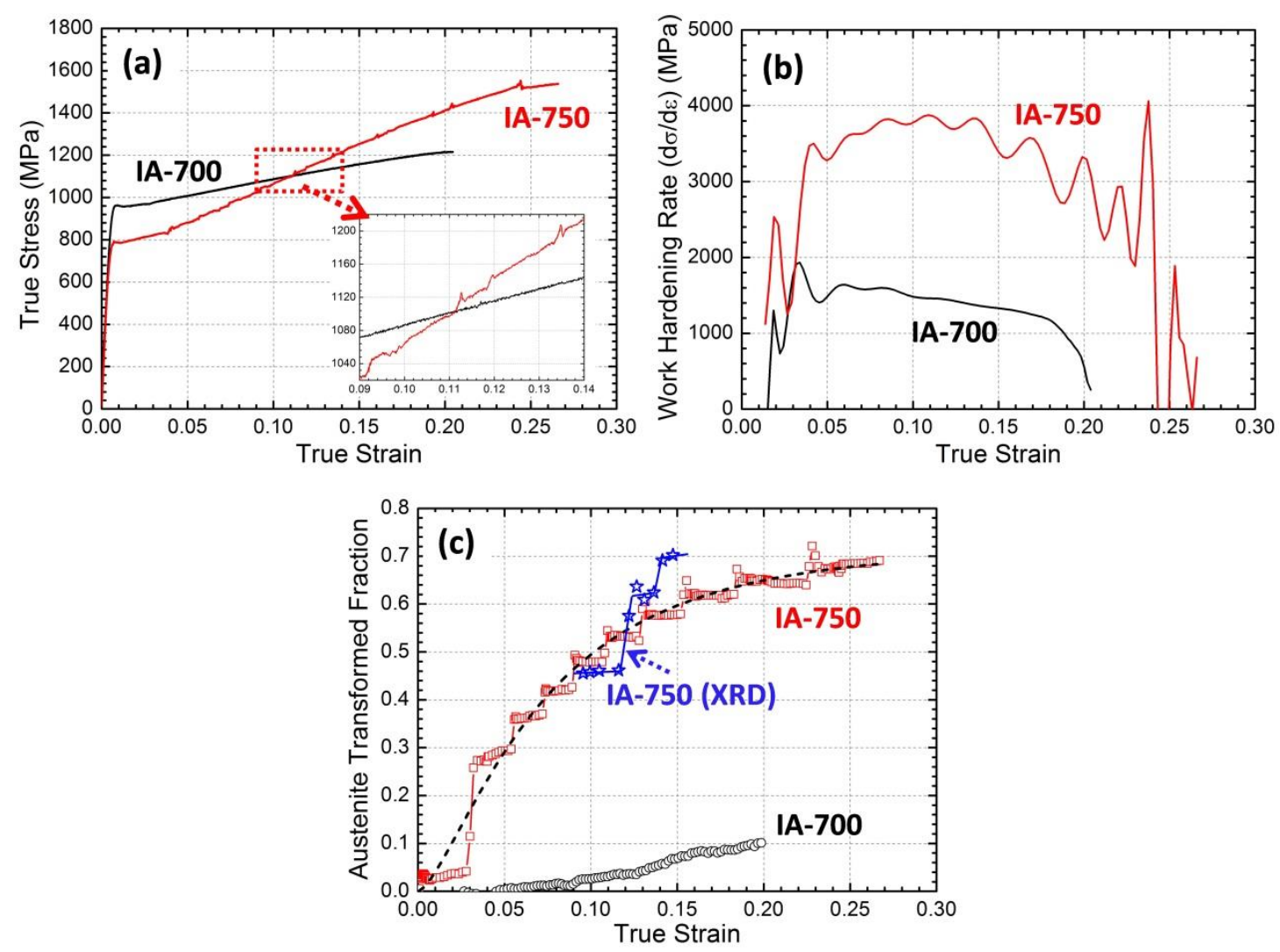

Fig. 2 (a) True stress-strain curve and (b) the rate of work hardening $(d \sigma / d \varepsilon)$ of the IA-700 and IA-750 sample; (c) Normalized austenite transformed fraction of the two samples during tensile straining, measured by Feritscope and XRD (the dash line is the fitting curve for the IA-750 sample based on the OC model). 

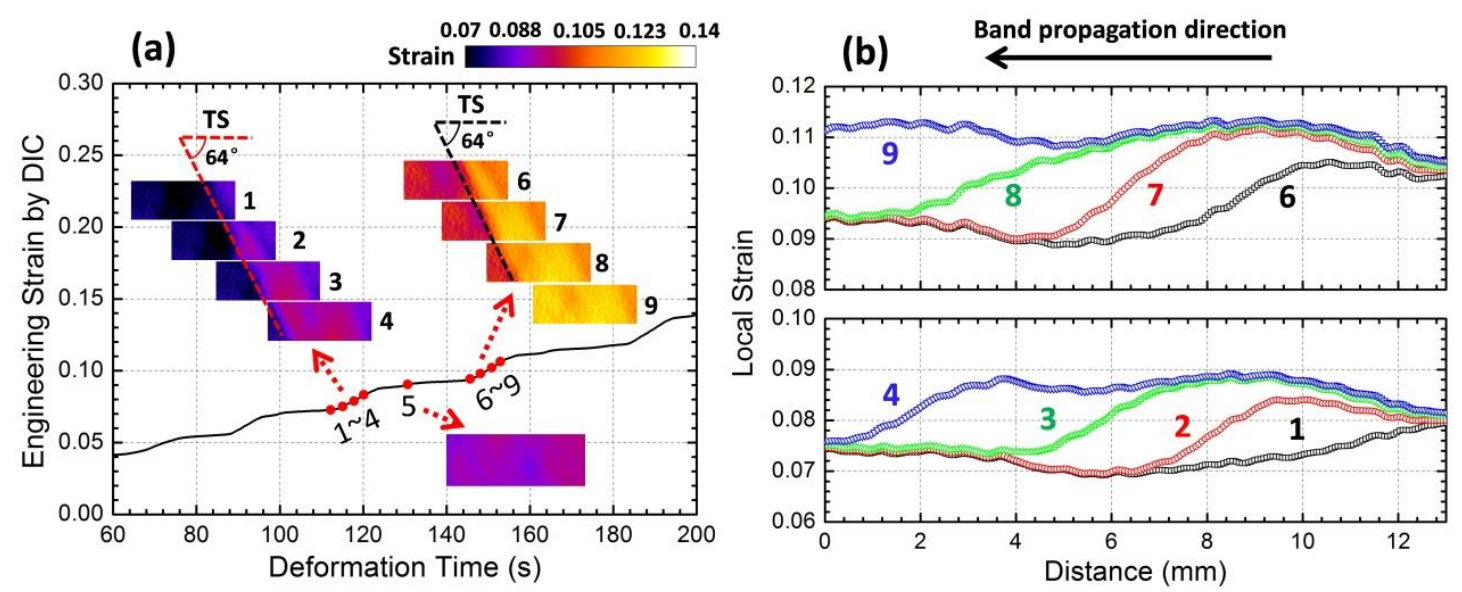

Fig. 3 (a) Average engineering strain generated by DIC as a function of deformation time and strain maps corresponding to the propagation of two successive bands in the IA-750 sample; (b) Corresponding strain profiles of the two bands along the tensile axis in the analyzed area.
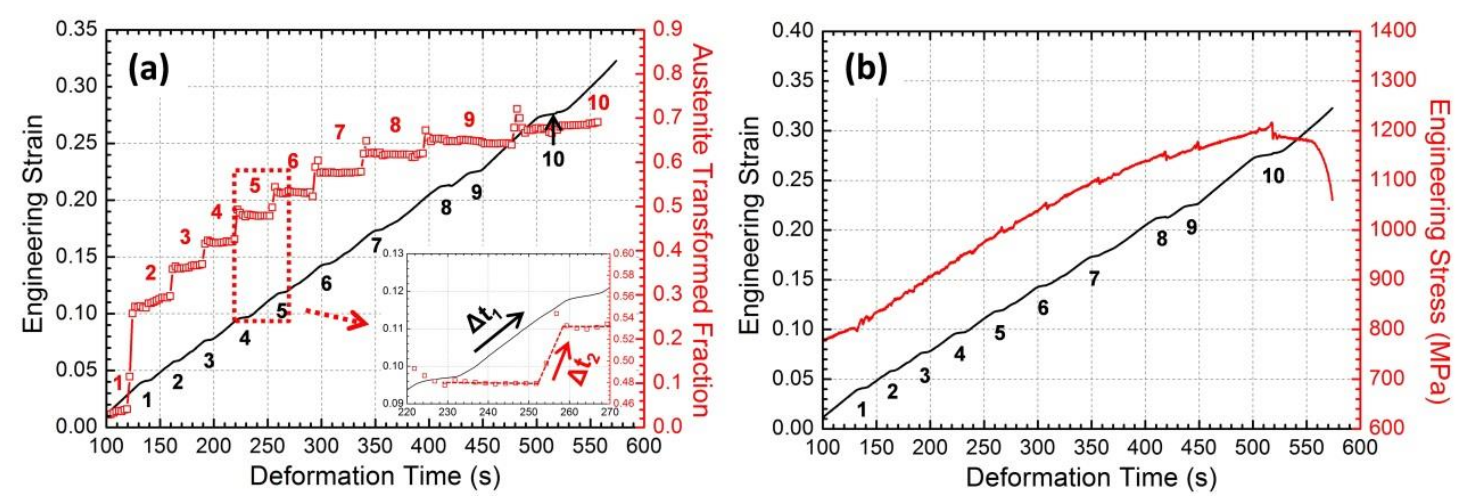

Fig. 4 Deformation time dependence of (a) engineering strain and normalized austenite transformed fraction and (b) engineering strain and stress for the IA-750 sample in the plastic deformation range. 